\title{
Theoretical Study of Gallium Phthalocyanine Dimer-Fullerene Complex for Photovoltaic Device
}

\author{
Akihiro Takeda ${ }^{1}$, Takeo Oku ${ }^{{ }^{*}}$, Atsushi Suzuki ${ }^{1}$, Yasuhiro Yamasaki ${ }^{2}$ \\ ${ }^{1}$ Department of Materials Science, The University of Shiga Prefecture, Hikone, Japan \\ ${ }^{2}$ Orient Chemical Industries, Co.Ltd., Department of New Business Sanra-Higashi-Machi, Neyagawa, Japan \\ E-mail:oku@mat.usp.ac.jp \\ Received February 14, 2011; revised April 24, 2011; accepted May 5, 2011
}

\begin{abstract}
Geometry and electronic structures of gallium phthalocyanine dimer and fullerene molecules were investigated by theoretical calculation. The highest occupied molecular orbital was localized on the donor site, and the lowest unoccupied molecular orbital were localized on accepter site. The present results indicate that high conversion efficiency would be expected when phthalocyanine dimers were used as solar cell materials.
\end{abstract}

Keywords: Phthalocyanine Dimer, Fullerene, Solar Cell

\section{Introduction}

Organic thin film solar cells are promising alternatives for low cost renewable energy, large-area fabrication on light weight, flexible substrates at low temperatures by coating and printing methods. Therefore, the organic solar cells are attractive for next generation solar cells. Metal phthalocyanines (MPc), which have photovoltaic property, heat-resistance, light-stability, chemical stability, and high optical absorption at visible range, are used for oxidation catalysts, catalysts of fuel cells and solar cells. An efficiency of $\sim 5 \%$ was achieved for organic solar cells by employing small molecules such as copper phthalocyanine and fullerenes [1-4]. When the nearest neighbor two phthalocyanines with substituent such as amino group and hydroxy group are connected by hydrogen bridged substituent, high photoconduction was observed [5]. However, few phthalocyanine dimers have been reported, and high photoconduction can be expected for the covalently-bridged phthalocyanine dimers.

The purpose of the present work is to investigate molecular orbital of gallium phthalocyanine $(\mathrm{GaPc})$ dimer and fullerene which is a good electronic accepter, and to examine this structure as solar cell material.

\section{Method of Calculations}

First principle calculations of the electronic properties have been used to predict the geometry of molecular structures and vibration spectra for phthalocyanine [6].
The optimized geometries and energies of all the present structures were calculated by ab-initio molecular orbital calculations using Gaussian 03. A detailed analysis of ground states of the electronic structures is based on selfconsistent solutions of the Kohn-Sham (KS) molecular orbital model on density functional theory (DFT) with hybrid B3LYP function [7]. The KS equation is defined as an Equation (1).

$$
\left[-\frac{1}{2} \nabla^{2}+\int \frac{\rho\left(r^{\prime}\right)}{\left|r-r^{\prime}\right|} \mathrm{d} r^{\prime}+\frac{\partial E_{x c}[\rho(r)]}{\partial \rho}+\gamma(r)\right] \varphi_{i}=\epsilon_{i} \varphi_{i}
$$

Exchange-correlation potential of the third term in the (1) is defined as the functional derivative of $E_{X C}[\rho(r)]$ with respect to $\rho(r)$. The isolated molecular structures were optimized by the DF calculation using restricted Hartree-Fock (RHF) and hybrid B3LYP function based on the Becke exchange function and the Lee-Yang-Parr correlation function, with the KS orbital expanded to LANL2DZ and STO-3G*, 3-31G* and 6-31G* basis sets. Electronic densities and energy gaps between highest occupied molecular orbital (HOMO) and lowest unoccupied molecular orbital (LUMO), and electronic densities were investigated.

\section{Results and Discussion}

Figure 1 is a structure of $\mu$-oxo-bridged gallium phthalocyanine dimer used in the present study. Two GaPc planes are parallel to one another, and the rotational de 


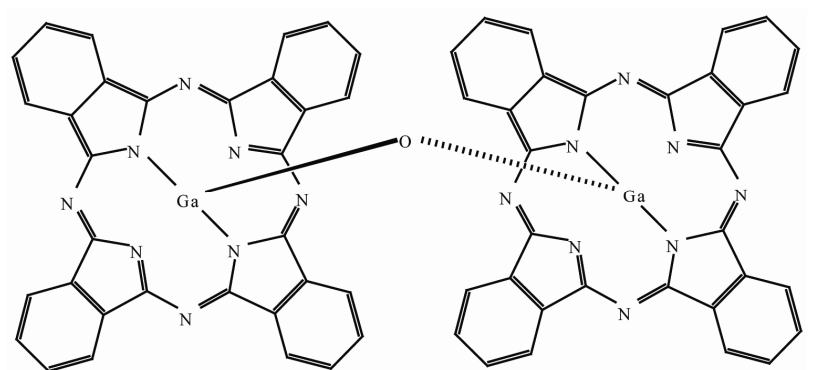

Figure 1. Structure of $\mu$-0xo-bridged gallium phthalocyanine dimer.

grees are $41.35^{\circ}$. The plane distance between GaPc monomer is $\sim 0.34 \mathrm{~nm}$ [8]. When the nearest neighbor two phthalocyanines is arranged with hydrogen bridged substituent, high photoconduction can be expected for the covalently-bridged phthalocyanine dimer.

Figure 2 shows HOMO and LUMO energy levels of the $\mathrm{GaPc}$ dimer with $\mathrm{C}_{60}$ after structural optimization using DFT/6-31G*. In Figure 2, electronic densities of

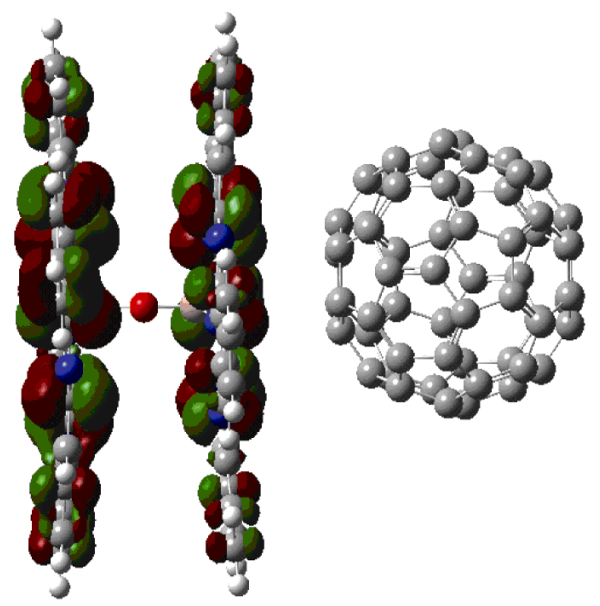

(a)
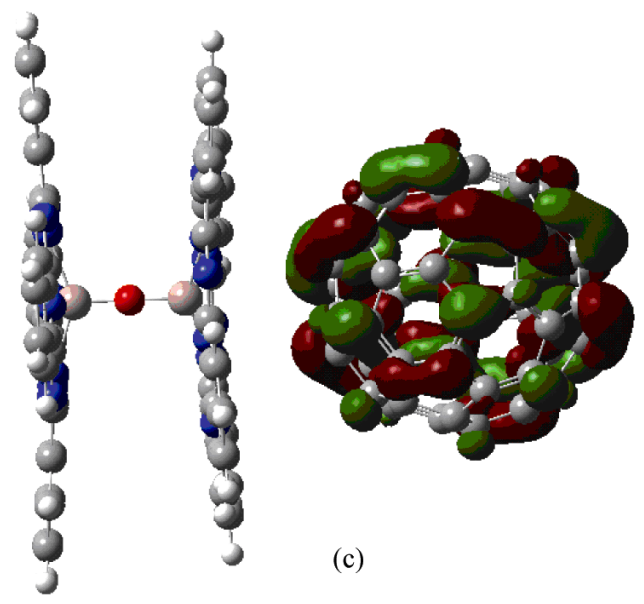

Figure 2. HOMO and LUMO energy levels of the GaPc dimer $+\mathrm{C}_{60}$. (a) HOMO, (b) LUMO, (c) LUMO+1, and (d) LUMO+2.
$\mathrm{LUMO}, \mathrm{LUMO}+1$, and LUMO+2 are localized for the fullerene side, while the HOMO is localized for the GaPc-dimer side, which suggests electron transfer between the GaPc dimer and fullerene. The similar localization of frontier orbital was previously reported for the other donor-fullerene supramolecular systems [9-11].

A schematic diagram of energy levels of GaPc dimer, $\mathrm{C}_{60}$, and $\mathrm{GaPc}$ with $\mathrm{C}_{60}$ is shown in Figure 3. The energy levels of the four highest occupied and four lowest unoccupied orbital levels are summarized in Table 1. It should be noted that the LUMO energy levels of the GaPc dimmer $+\mathrm{C}_{60}$ are comparable to the LUMO energy levels of fullerene, and the HOMO energy levels of the GaPc dimer $+\mathrm{C}_{60}$ are close to the HOMO energy levels of $\mathrm{GaPc}$ dimer. However, the symmetry of the GaPc dimer seems to be lowered because of decreasing of degeneracy, which would be due to the interaction with $\mathrm{C}_{60}$.

Electronic density of the GaPc dimer was widely dis-
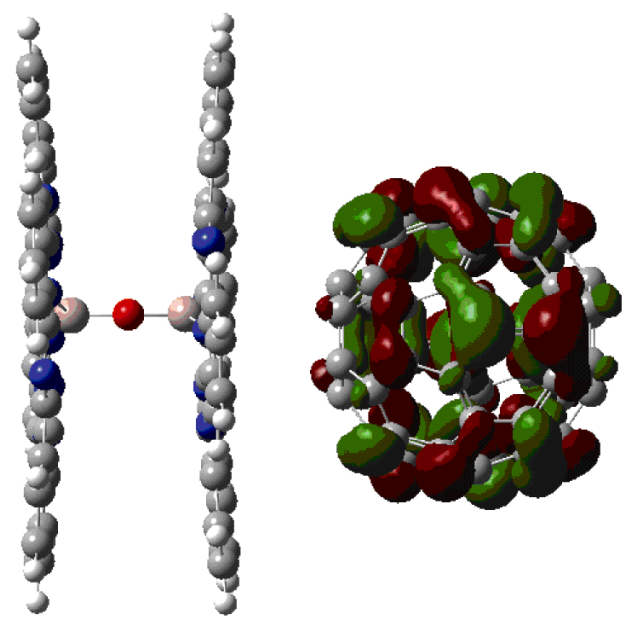

(b)
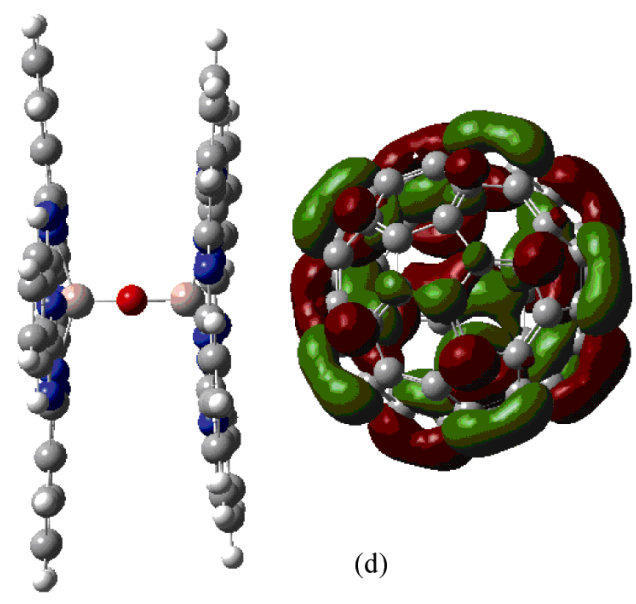

(d) 


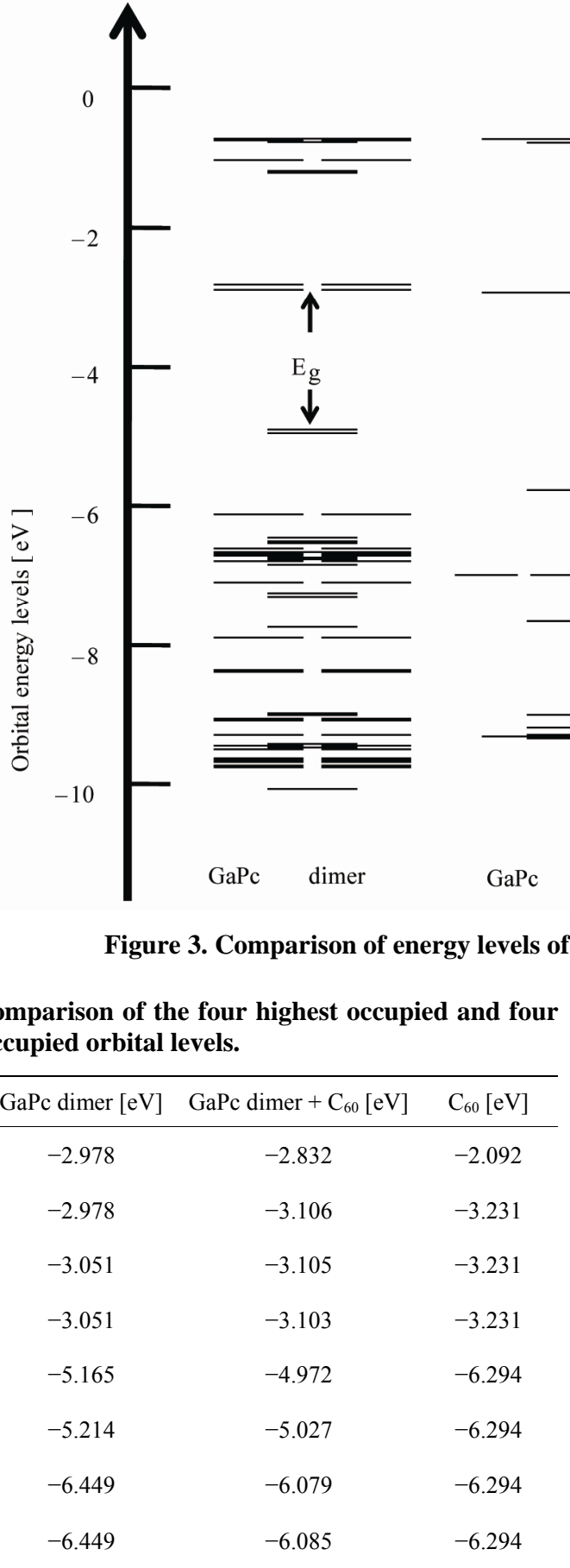

tributed around $\pi$ orbital on the both aromatic rings as observed in Figure 2(a). Energy levels of GaPc dimer at HOMO and LUMO were calculated to be $-5.165 \mathrm{eV}$ and $-3.051 \mathrm{eV}$, as listed in Table 1. The energy level of GaPc dimer would indicate advantage of GaPc dimer for charge-transfer of the excited carriers to conduction band of $\mathrm{C}_{60}$. The HOMO of GaPc dimer and the LUMO of $\mathrm{C}_{60}$ is closely related with open circuit voltage $\left(\mathrm{V}_{\text {oc }}\right)$ of the solar cells, which is described by $\mathrm{V}_{\mathrm{oc}}=(1 / \mathrm{e})\left(\left|\mathrm{E}_{\mathrm{GaPc}} \mathrm{HOMO}\right|\right.$ - $\left.\left|\mathrm{E}_{\mathrm{C} 60} \mathrm{LUMO}\right|\right)-0.3(\mathrm{~V})$, where $\mathrm{e}$ is the elementary change [12]. The value of $0.3 \mathrm{~V}$ is an empirical factor and this is enough for efficient charge separation $[13,14]$. The model of photovoltaic mechanism would be described by this equation, and the control of the energy levels is important for improving conversion efficiency. It is believed that the present $\mathrm{GaPc}$ dimer would be useful for the solar cells as previously reported fullerenebased supramolecules for high-performance photovoltaic devices [10], and excitation and charge transfer during light irradiation should be investigated further.

\section{Conclusions}

Geometry and electronic structure of phthalocyanine dimer-fullerene molecules were investigated by using $a b$-initio molecular orbital calculations, which indicated that the HOMOs were localized on the donor site and LUMOs were localized on accepter site. The present $\mathrm{GaPc}$ dimers would be expected as solar cell materials.

\section{Acknowledgements}

The authors would like acknowledge Prof. Mizuseki for 
useful discussion.

\section{References}

[1] F. Yang, M. Shtein and S. R. Forrest, "Morphology Control and Material Mixing by High-Temperature Organic Vapor-Phase Deposition and Its Application to Thin-Film Solar Cells," Journal of Applied Physics, Vol. 98, No. 1, 2005, pp. 1-10. doi:10.1063/1.1941480

[2] J. Xue, S. Uchida, B. P. Rand and S. R. Forrest, "Asymmetric Tandem Organic Photovoltaic Cells with Hybrid Planar-Mixed Molecular Heterojunctions," Applied Physics Letters, Vol. 85, No. 23, 2004, pp. 1-3. doi:10.1063/1.1829776

[3] N. Li, B. E. Lassiter, R. R. Lunt, G. Wei and S. R. Forrest, "Open Circuit Voltage Enhancement Due to Reduced Dark Current in Small Molecule Photovoltaic Cells," Applied Physics Letters, Vol. 94, No. 2, 2009, pp. 1-3. doi:10.1063/1.3072807

[4] B. P. Rand, J. Xue, S. Uchida and S. R. Forrest, "Mixed Donor-Acceptor Molecular Heterojunctions for Photovoltaic Applications. I. Material Properties," Journal of Applied Physics, Vol. 98, No. 12, 2005, pp. 1-7. doi:10.1063/1.2142072

[5] Y. Yamasaki, K. Kuroda and K. Takaki, "Synthesis of New Polymorphs of M-Oxo-Metal(III) Phthalocyanine Dimmers and Their Photoconductive Properties," Journal of the Chemical Society of Japan, Vol. 1997, No. 12, 1997, pp. 887-898.

[6] D. Li, Z. Peng, L. Deng, Y. Shen and Y. Zhou, "Theoretical Studies on Molecular Structure and Vibrational Spectra of Copper Phthalocyanine," Vibrational Spectroscopy, Vol. 39, No. 2, 2005, pp. 191-199. doi:10.1016/j.vibspec.2005.03.004

[7] W. Kohn and L. J. Sham, "Self-Consistent Equations Including Exchange and Correlation Effects," Physical Review, Vol. 140, No. 4A, 1965, pp. 1133-1138. doi:10.1103/PhysRev.140.A1133
[8] A. Takeda, A. Minowa, T. Oku, A. Suzuki, K. Kikuchi and Y. Yamasaki, "Formation and Characterization of Phthalocyanine Dimer/C 60 Solar Cells," Progress in Natural Science: Materials International, Vol. 21, No. 1, 2011, pp. 27-30.

[9] H. Mizuseki, N. Igarashi, R. V. Belosludov, A. A. Farajian and Y. Kawazoe, "Theoretical Study of Phthalocyanine-Fullerene Complex for a High Efficiency Photovoltaic Device Using Ab Initio Electronic Structure Calculation," Synthetic Metals, Vol. 138, No. 1-2, 2003, pp. 281-283. doi:10.1016/S0379-6779(02)01289-4

[10] H. Mizuseki, N. Igarashi, R. V. Belosludov, A. A. Farajian and Y. Kawazoe, "Theoretical Study of Chlorin-Fullerene Supramolecular Complexes for Photovoltaic Devices," Japanese Journal of Applied Physics, Vol. 42, No. 4B, 2003, pp. 2503-2505. doi:10.1143/JJAP.42.2503

[11] A. J. Hameed, "Theoretical Investigation of a Phthalocyanine-Fulleropyrrolidine Adduct and Some of Its Metallic Complexes," Journal of Molecular Structure: THEOCHEM, Vo. 764, No. 1-3, 2006, pp. 195-199. doi:10.1016/j.theochem.2006.02.021

[12] M. C. Scharber, D. Mühlbacher, M. Koppe, P. Denk, C. Waldauf, A. J. Heeger and C. J. Brabec, "Design Rules for Donors in Bulk-Heterojunction Solar Cells-Towards 10\% Energy-Conversion Efficiency," Advanced Materials, Vol. 18, No. 6, 2006, pp. 789-794. doi:10.1002/adma.200501717

[13] R. Ulbricht, S. B. Lee, X. Jiang, K. Inoue, M. Zhang, S. Fang, R. H. Baughman and A. A. Zakhid, "Transparent Carbon Nanotube Sheets as 3-D Charge Collectors in Organic Solar Cells," Solar Energy Materials and Solar Cells, Vol.. 91, No. 5-6, 2007, pp. 416-419. doi:10.1016/j.solmat.2006.10.002

[14] T. Oku, T. Noma, A. Suzuki, K. Kikuchi and S. Kikuchi, "Fabrication and Characterization of Fullerene/Porphyrin Bulk Heterojunction Solar Cells," Journal of Physics and Chemistry of Solids, Vol. 71, No. 4, 2010, pp. 551-555. doi:10.1016/j.jpcs.2009.12.034 